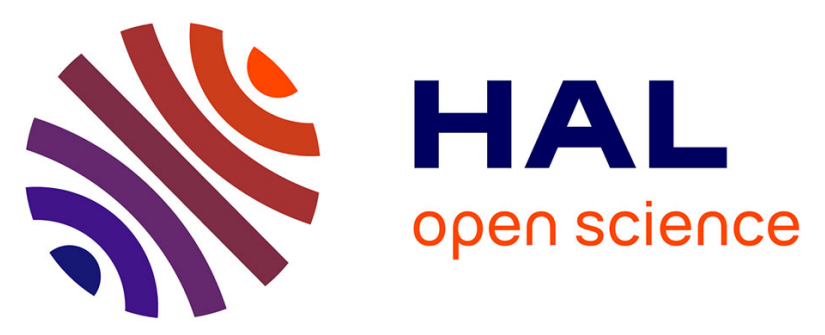

\title{
A Collaborative Cyber-Physical Microservices Platform - The SITL-IoT Case
}

Carlos Gonçalves, A. Luis Osório, Luis Camarinha-Matos, Tiago Dias, José

Tavares

\section{- To cite this version:}

Carlos Gonçalves, A. Luis Osório, Luis Camarinha-Matos, Tiago Dias, José Tavares. A Collaborative Cyber-Physical Microservices Platform - The SITL-IoT Case. 22nd Working Conference on Virtual Enterprises (PRO-VE 2021), Nov 2021, Saint-Etienne, France. pp.411-422, 10.1007/978-3-030-859695_38. emse-03345991

\section{HAL Id: emse-03345991 https://hal-emse.ccsd.cnrs.fr/emse-03345991}

Submitted on 25 Nov 2021

HAL is a multi-disciplinary open access archive for the deposit and dissemination of scientific research documents, whether they are published or not. The documents may come from teaching and research institutions in France or abroad, or from public or private research centers.
L'archive ouverte pluridisciplinaire HAL, est destinée au dépôt et à la diffusion de documents scientifiques de niveau recherche, publiés ou non, émanant des établissements d'enseignement et de recherche français ou étrangers, des laboratoires publics ou privés. 
Gonçalves C., Osório A.L., Camarinha-Matos L.M., Dias T., Tavares J. (2021) A Collaborative Cyber-Physical Microservices Platform - the SITL-IoT Case. In: Camarinha-Matos L.M., Boucher X., Afsarmanesh H. (eds) Smart and Sustainable Collaborative Networks 4.0. PRO-VE 2021. IFIP Advances in Information and Communication Technology, vol 629. Springer, Cham. https://doi.org/10.1007/978-3030-85969-5_38

\title{
A Collaborative Cyber-Physical Microservices Platform - The SITL-IoT Case
}

\author{
Carlos Gonçalves $^{1}$, A. Luís Osório ${ }^{1}$, Luís Camarinha-Matos², Tiago Dias ${ }^{1}$, José \\ Tavares $^{3}$ \\ ${ }^{1}$ ISEL - Instituto Superior de Engenharia de Lisboa, IPL - Instituto Politécnico de Lisboa, \\ and POLITEC\&ID, Portugal, carlos.goncalves@isel.pt, lo@isel.ipl.pt, tiago.dias@isel.pt \\ ${ }^{2}$ School of Science and Technology, NOVA University of Lisbon and CTS-UNINOVA, \\ Portugal, cam@uninova.pt \\ ${ }^{3}$ FORDESI, Informatics Systems, and Solutions Company, jose.tavares@,fordesi.pt
}

\begin{abstract}
Managing heterogeneous software and hardware artifacts from multiple suppliers is a complex and challenging process. The integration of sensors, actuators, and their controllers, modeled as IoT elements, also presents significant challenges. Typically, a vendor supplies one or more parts, each one with its proprietary interface, which may raise vendor lock-in and supplier dependencies that can compromise the replacement of some of the artifacts by equivalent ones from competing vendors. The research presented in this paper addresses such challenges in the context of the SITL-IoT project aiming at transforming an industrial agri-food environment towards an open, integrated system-of-systems. We present and discuss a reference implementation of a collaborative platform to simplify the management of different artifacts, supplied by alternative suppliers, modeled as services. More specifically, the concepts of ISystem (Informatic System), CES (Cooperation Enabled Service), and Service are used to manage the different elements that compose an agrifood environment transparently and uniformly. We argue that the adopted model simplifies the collaboration among technology suppliers along the life cycle maintenance and evolution of their enabled products.
\end{abstract}

Keywords: Internet of Things, Systems Integration, Collaborative Networks, Cyber-physical systems, Microservices, Distributed systems.

\section{Introduction}

Organizations that use different software or hardware elements face challenging problems when updating or upgrading their technological infrastructures. Typically, each technology solution or product is provided by a different supplier with its own proprietary protocols, which quite often makes it very difficult and expensive to replace a given element with an equivalent one from a competing supplier. On the other hand, the Internet of Things (IoT) enables industries to manage their existing sensors and actuators as elements that exist on their local networks or WAN. However, because collaborating suppliers deliver sensors and actuators using different protocols and Application Programming Interfaces (APIs), the integration of 
such technology artifacts results in complex and demanding processes both in terms of the development and maintenance cycles. Indeed, competing suppliers source elements under technology diversity, raising risks of vendor lock-in or supplier dependencies. Such dependencies compromise the replacement of artifacts, being an obstacle to sustainable innovation.

This paper presents and discusses a reference implementation of an Informatics System of Systems (ISoS) platform [8] that contributes to the Model-Driven Open Systems Engineering (MDEOS) and promotes an open market competitive technology landscape for organizations. The ISoS model establishes a system-ofsystems where each system might have market competitors able to provide possible substitutions. The main objective is to make a system, or elements of a system, replaceable by an equivalent technology artifact from an alternative supplier. The notion of Cooperation Enabled Services (CES) is adopted as part of the strategy to attain partial substitutability, a challenging endeavor to achieve. The ISoS model comprises three abstraction layers: i) ISystem, establishing a coarse computational and cooperation responsibility border; ii) CES, as a composite of Services; and iii) Service, as the operating element that can be a pure software artifact or a cyberphysical element, e.g., an IoT sensor/actuator, as the finer-grained computational responsibility border. By ISoS reference implementation, we mean the instantiation of an operating ISystem, named ISystemo, aiming to validate and certify the compliance of all the ISystem/CES/Service products.

This work expands further the initial approach of the SITL-IoT project [12], aiming to evolve an industrial agri-food environment towards an agri-food ecosystem supported by an open, integrated system-of-systems. We present the first ISoS reference implementation and discuss its utilization for simplifying the management of artifacts supplied by alternative vendors. Such ISoS reference implementation is the first effort to deliver an actual implementation of the ISoS model, thus allowing organizations to be ISoS enabled. As a case study, we demonstrate the ISystem, CES, and Services instances developed within the SITL-IoT project devoted to structure and manage the agri-food silos environment transparently and uniformly.

The remainder of this paper is organized as follows. Section 2 briefly presents the ISoS background, while Section 3 reviews the SITL-IoT project and its strategies to integrate the ISoS reference implementation. Finally, Section 4 presents the conclusions and discusses future work.

\section{Enterprise Architecture with ISoS Background}

By adopting the ISoS framework [8], an enterprise platform architecture is based on three core modeling elements: ISystem, CES, and Service. Furthermore, to be ISoS enabled, an organization needs to instantiate the meta-ISystem, i.e., an instance of the ISystem, ${ }_{0}$ an ISystem with the unique role of managing the ISoS landscape. Fig. 1 depicts the primary elements that make an ISoS organization using a SysML Block Definition Diagram. The ISoS abstraction is a composite of exactly one ISystem ${ }_{0}$ and zero or more ISystems. Each ISystem is composed of one or more CES, which are composed of one or more Services. The ISoS elements model the technology artifacts 
through a set of properties, e.g., name, version, supplier, or description. In the case of a Service, the modeling element instance has associated the meta-data required for a peer Service to access the implemented functionalities.

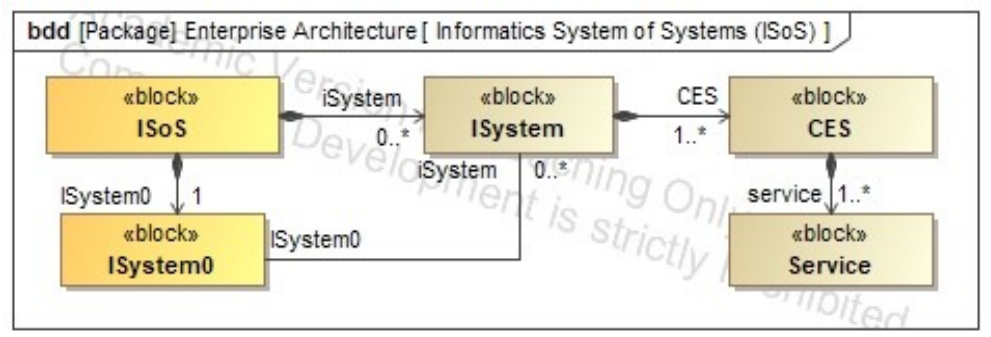

Fig. 1. The simplified SysML block definition diagram of the ISoS model

The ISoS model considers a meta-element with management or coordination roles at the ISoS, ISystem, and CES levels, respectively ISystem ${ }_{0}, C E S_{0}$, and Service. A primary role of the $I_{\text {System }}$ is to act as a directory service managing the metadata of the ISoS elements that exist within an organization. In the current version of the ISoS reference implementation, the ISystem 0 relies on Apache Zookeeper [4]. Fig. 2 depicts the internal structure of the ISystem 0 linked to the ISoS Znode, the children nodes ISystem $_{0}, I_{\text {ISystem }}, \ldots$, ISystem $_{N}$, the corresponding children CES, and, for each $C E S_{J}$, the children Services. ISystem 0 has a CES 0 composed by Ser 0 and Ser $_{l}$. The ISoS administration user interface has a $C E S_{U I}$ composed of $\operatorname{Ser}_{0}$ and $\operatorname{Ser}_{U I}$ that makes possible the navigation across ISoS instance elements, facilitating introspection of its properties.

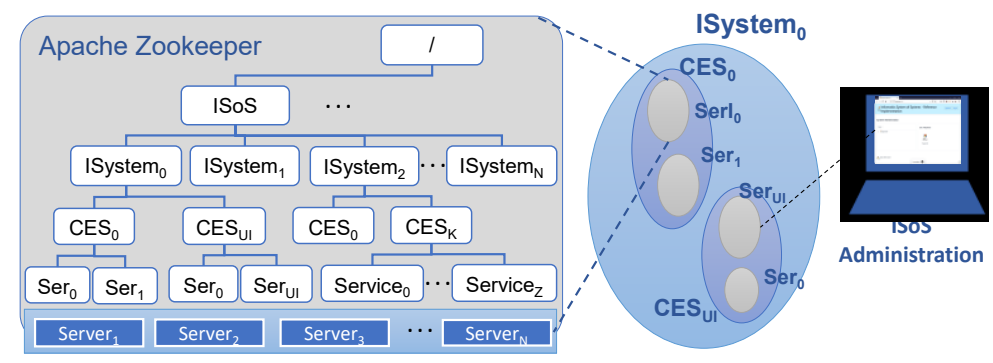

Fig. 2 The internal organization of the ISystemo

The adoption of the open-source Zookeeper system is motivated by ISystem 0 being a critical system since the other ISystems depend on its availability. If configured in redundancy mode, the Zookeeper system maintains a consistent replica in $\mathrm{N}$ independent servers, preferably based on separate hardware. The approach follows the strategy proposed in [10], considering a reliable ISystem $_{0}$ dependent on the faulttolerant configuration of the Zookeeper, implementing the Zab distributed coordination algorithm [3], [4]. Furthermore, beyond the fault-tolerance and distributed coordination strategies [6], the ISystem 0 implementation is prepared to 
scale several Service instances through the Observer nodes concept to speed up readonly service lookup operations [5].

One important feature of the ISoS is its capability to make any Service instance accessible both inside and outside the organization. The ISystem 0 is accessible at isos.organizationDomain:2058. Business partners, such as a technology supplier of an ISystem, a CES, or a Service, can use this access to collaborate in the maintenance or evolution of the supplied technology artifacts. The access facility offered by ISoS, through ISystemo, is accessible in any business collaboration context by following the appropriate authentication and security mechanisms. In the next section, we detail implementation issues of the reference ISystem $_{0}$ developed in the SITL-IoT project with further contributions from [12].

\section{The SITL-IoT Project Case Study}

The SITL-IoT research and development project aims at developing an open IoT Bus for cyber-physical elements modeled as Services. The project answers the research question of how to evolve towards an open multi-supplier technology landscape. In this section, we show how the ISoS model was used to structure the computing elements that compose the SITL-IoT project.

\subsection{The SITL-IoT Base Scenario}

Fig. 3 depicts a simplified view of an agri-food company located nearby the seaport of Leixões in the north of Portugal, identified as Organization A.
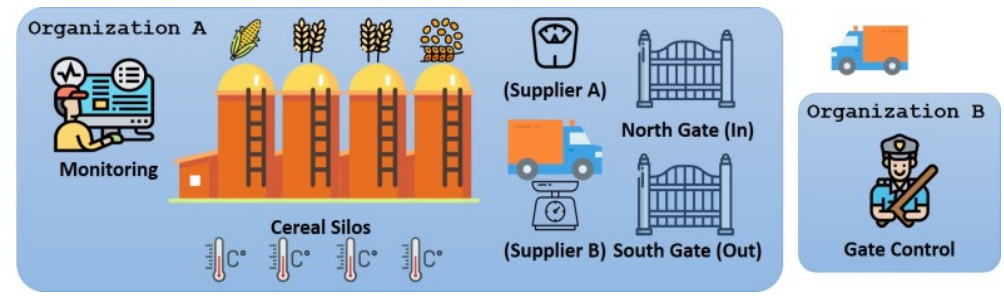

Fig. 3. Case study scenario

In this scenario, we consider only a subset of the elements necessary for loading and unloading cereals to/from trucks for reasons of simplicity. For truck control, access to the industrial facilities is done using two gates: North for inbound and South for outbound. Moving agri-food barges inside the seaport requires an authorization issued by the Port Authority, represented by Organization B. The purpose of the gate in Organization B is to control the trucks discharging the bulk-carrier ships from the seaport area. This area is the Portuguese and EU border with customs and borderpolice control. As such, the movement of products between the seaport and the agrifood organization requires drivers to authenticate and validate its transport. As shown in Fig. 3, trucks are weighted both inbound and outbound using industrial scales from 
different suppliers with its own specific weigh controller technology and interfaces. The silos include several temperature sensors that are used to manage the temperature at regularly spaced levels of its structure. This weighing bridge infrastructure, the temperature sensor elements, and other cyber-physical systems of Organization A are modelled as IoT devices. Each IoT device is a Service element of the ISoS framework. All ISystem, CES, and Service elements may have an associated synoptic panel for the monitoring and operating of the physical elements. For the visualization of interrelated technology elements, from ISystems to Service, a generic Synoptics of Things framework is being developed to simplify central supervision interfaces [13].

\subsection{The SITL-IoT Project Structure and Elements}

The ISoS reference implementation groups the artifacts into specialized projects as Application Programing Interface and Model Elements (APIM), Operations Elements (OPE), Deployment and Operations Elements (DOE), and Monitoring Elements (MOE). This approach aims to facilitate the integratation of complex technology landscapes, complying to the reference structure and following the guidelines suggested by the Collaborative Enterprise Development Environment (CEDE) [7]. Fig. 4 shows the ISoS reference implementation structure with the ISystem 0 and the corresponding CES and Service elements. The elements ISystem and CES are organized using the above-mentioned specialized projects (modules) DOE and MOE, since the APIM and OPE are exclusive of the Service elements.

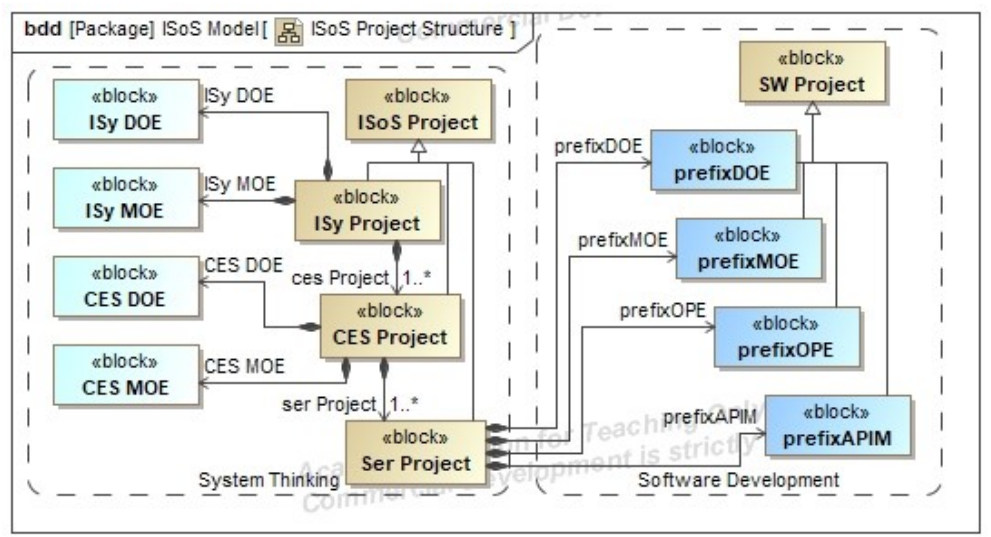

Fig. 4. ISoS Reference Implementation project structure (CEDE concerns)

The DevOps approach inspires the DOE project incorporating the mechanisms to coordinate the development and instantiation of executive parts of ISoS [1]. The MOE aims to deal with the monitoring mechanisms, e.g., by adopting the Simple Network Management Protocol (SNMP) with the respective Management Information Base (MIB) to model the instrumentation of Service elements. The technology selection can also use the Java Management Extension (JMX) protocol and the respective instrumentation modeling using Mbean to be managed by JMX agents. The 
monitoring of technology artifacts is of paramount importance to achieve reliable integrated systems, as discussed in the ISoS framework reliability [11]. OPE organizes the computational logic making a Service entity. The project of a Service also includes: i) the APIM module, to define interfaces and models specific to the Service; ii) the MOE module, to support the implemented monitoring elements; iii) the DOE module, responsible for deploying the Service. An alternative is to associate the CES DOE module responsible for deploying the composing Service elements. Another option is to consider an integrated deployment of an ISystem done by its DOE project element. In the current ISystem deployment, the strategy is to invoke the DOE projects of the ISystem or CES composites recursively. For each CES, the element executes the deployment logic until the leaf Service elements.

The ISoS reference implementation was developed based on the Java ecosystem, using Apache Maven to structure the project, manage the dependencies, and generate isolated and composed artifacts. Nevertheless, very similar principles can be used to develop an ISoS reference implementation using any other technological ecosystem.

As discussed in [7], while ISoS aims to contribute to the substitutability of technology artifacts (Service, CES, or ISystem), technology independence needs to be completed by a unified development environment for unique technology artifacts. Accordingly, the ISoS reference implementation establishes a separation between ISystems, CES, and Service elements as concepts, what we refer to as system thinking to enforce technology independence. The realization of Services in some technology and executed within the organization (on-premises) or on the cloud refers to software and deployment/management issues. Fig. 4 depicts System Thinking and Software Development dotted boxes. The System Thinking dotted box represents the ISoS ISystem, CES, and the Service concept as system elements. The Software Development box represents the software and integration issues considering the required technology artifacts making the Service an executable entity.

As presented and discussed in section 2, the ISystemo primarily acts as the ISoS directory service of an organization (to locate Service technology elements). Thus, depending on the current state of Service (Deployed, Running, Undeployed, Restarting, Shutting-down, etc.), such state is reflected in the administration interface of the ISystem 0 . The diversity of technologies and strategies to address the DevOps approach, e.g., Ansible, and Kubernetes (container orchestration), motivated a comparative study for a continuous architecting with Microservices and DevOps [14]. Our reference implementation aims to make the ISystem 0 a governance platform generalized to manage the life cycle of Service concept instances and their containment structures (CES and ISystems). Since a Service exists in the context of a $C E S$ and a $C E S$ exists in the context of an ISystem, we can consider the registering of a Service within the ISoS landscape involving the following steps:

a. Create (or update) the meta-information of the corresponding ISystem;

b. Create (or update) the meta-information of the corresponding CES(s);

c. Create (or update) the meta-information of the Service(s);

d. Start the OPE and MOE modules of the Service(s).

As a result, the ISoS reference implementation includes i) a generic ISystem DOE capable of implementing step a; ii) a generic CES DOE capable of implementing step $\mathrm{b}$; and iii) a generic Service DOE capable of implementing steps $\mathrm{c}$ and $\mathrm{d}$. Furthermore, since in this case study the Java ecosystem was used as the base for the 
ISoS reference implementation, the above artifacts are made available as independent JAR files. This approach allows us to change the implementing artifact by a competing one (substitutability). Although the discussion presented in this work is focused on the Java ecosystem, the proposed concepts can be extended to other ecosystems. In fact, that extension can be a very straightforward process that consists only in the configuration of the above mentioned JAR files to execute native Operating System processes rather than Java processes.

For an ISystem reference implementation, the DOE project module is a Java command-line tool (CLI) that receives two XLM files as arguments. The ISystem metadata is specified with the argument $-d$ isystemDef. $x m l$. The list of configuration elements used to start all the CES included in this ISystem is set with the argument -c cesCfg.xml. Each configuration element has the location of: i) the CES DOE module; ii) the file containing the CES metadata; and iii) the file containing the configuration of the services included in the CES. All file paths in the configuration elements are relative to a base directory, specified as an attribute in the configuration file. Additionally, the configuration file has two attributes to specify the path of the Java Virtual Machine (JVM) and the base working directory of the modules to start.

The CES reference implementation considers that the DOE module follows a similar approach to the one used in the ISystem DOE module. It is a Java CLI application that receives as arguments the name of the XML file containing the metadata of the CES (-d cesDef. $x m l$ ) and the name of the XML file containing the configuration of the Service elements that compose the CES (-c serviceCfg.xml).

The file used to define the Service configuration has all the information to start a Service, including the DOE, OPE, and MOE modules and the corresponding arguments. Please note that the OPE and MOE modules are the only ones committed to specific functionalities, represented using a darker blue in Fig. 4. The ISoS reference implementation offers a default DOE module, assuming that the OPE and MOE Service modules are JAR files receiving their arguments in the command line.

\subsection{The ISoS Administration User Interface for the SITL-IoT Case}

An administrator can use the ISoS user administration interface to register the different Services that compose the ISoS landscape organization using only the OPE and MOE modules of each Service and a set of configuration files, as discussed in the previous section. The fulfillment of the ISoS interface with the tree ISoS/ISystem/CES/Service is, therefore, a quite straightforward task, as a result of the reference implementation discussed in the previous sections.

It is worth mentioning that advanced abstractions are under evaluation, namely the use of container orchestrations, e.g., the Kubernetes automated container deployment, scaling, and management toolset. However, our approach does not aim exclusively for the cloud. In fact, we strive for a balanced strategy for the organization's computing technology landscape that can be deployed either on-premises or on the cloud, depending on resource allocation needs and the most advantageous options that can change dynamically. The vendor lock-in risks motivated the proposal of a "... overlay layer that provides users with an inter-operable and visibility-supported environment 
for MSA-based IoT-Cloud service composition over the existing multiple clouds" [2]. Nonetheless, the proposed layer seems to introduce additional complexity. The DOE project structuring element can manage the deployment issues in our approach, eventually providing alternative implementations to cope with cloud provider's heterogeneity.

\subsection{Revisiting the SITL-IoT Scenario under a Collaborative Perspective}

The ISoS implementation described in the previous sections also enables to analyze the SITL-IoT scenario presented in section 3.1 under the collaborative network perspective. As discussed above, every time a truck needs to enter the agri-food area located on-premises of Organization A, it is necessary to obtain inbound access issued by Organization B. Using the ISoS model and its associated reference implementation, the collaboration between the two organizations is a straightforward process. Each of the gates shown in Fig. 3 is running a Service, denoted as ServiceA, performing the following actions:

1. Collect the driver and truck identification;

2. Contact the ISoS landscape of Organization B (isos.organizationB:2058) to get an instance of its ISystem, denoted as $\operatorname{ISystem}_{0} B$;

3. Using ISystem ${ }_{0} B$, ServiceA performs a lookup operation to obtain the Service responsible for granting the entry access, denoted as ServiceGateB;

4. ServiceA uses ServiceGateB to authenticate the driver and the truck;

5. If the authentication is successful, the truck can access the agri-food area.

This simple example shows that the presented ISoS reference implementation allows establishing collaboration among two different organizations, each with wellidentified responsibilities, without knowing the internal details of the involved organizations. However, the example can be extended to more complex scenarios involving several organizations. The only requirement is that the involved organizations can access the ISoS landscape of each other, i.e., access the involved ISystem $_{0}$. One main problem is that for ServiceA of Organization A to access ServiceGateB of Organization B, there is a need for ServiceA to know a priori the path ISystem $_{i} /$ CES $_{j} /$ ServiceGateB and with it obtain the ServiceGateB meta-data. This problem can be resolved using ISoS. With the ServiceGateB metadata, the ServiceA client from Organization A can get the necessary data to configure the client proxy to access the implemented functionalities properly.

The collaboration infrastructure offered natively by the ISoS framework can be enhanced by adopting the ECoNet collaborative infrastructure [9]. In this case, ServiceA of Organization A used its ECoM ISystem to have access to a collaboration context shared with Organization B, that provides the required interaction with ServiceGateB using an ECoM instance in Organization B. The advantage of collaboration through the ECoM ISystems is that domain application ISystems share low-level communication, security mechanisms, and higher-level virtual collaboration contexts multi-tenant groups. 


\section{Conclusions and Further Research}

This paper presents and discusses a reference implementation of the ISoS framework, which models the computing technology landscape of an organization. The Java ecosystem adopting the Apache Zookeeper and other open-source projects supports the validation of the framework in the context of the SITL-IoT project. Beyond the ISystem $_{0}$ as a core technological element for any ISoS enabled organization, we present and discuss a project structure to avoid dependency from subcontracted developments. Furthermore, we discuss a modeling schema for the automatic management of ISoS concept instances. Also, we demonstrate how this approach enables configuring operating system services to automatically register an ISoS Service and the corresponding ISystem and CES when the computer (physical or virtual) supporting the Service's execution starts.

We further discuss a monitoring strategy based on SNMP agents operationalized by the ISoS Service concept and managed by the MOE project structuring element. The association of monitoring Service agents to domain application Services requires further research considering the need to abstract legacy protocols, following the adaptive ISoS Service interoperability mechanism.

For software solution providers like Fordesi, ISoS is a tool that brings industrial IoT solutions to the transport and logistics sector. The modularity and decoupling strategies used by the framework enables a quick-wins project management approach that leads to time and cost-effective solutions.

Concerning collaboration issues, the proposed approach based on the ISoS framework offers collaboration support facilities, since services from collaborative organizations can mutually find each other and interoperate based on the $I_{0}$ canonical entry point and ISoS metadata facilities. In addition, we discuss the alternative ECoNet using the collaborative contexts and virtual collaboration contexts as shared infrastructure elements. While the collaboration mechanisms offered by ISoS proved to be sufficient for the current business case, further research will validate the adoption of ECoNet infrastructure as a more general approach.

Acknowledgments. The research conducted by GIATSI/ISEL/IPL was developed in collaboration with the SOCOLNET scientific network and its ARCON-ACM initiative. The ANSR/SINCRO, BP/HORUS, and FORDESI/SITL-IoT-PT-2020 projects partially supported this research. Partial support also from the Center of Technology and Systems - UNINOVA, and the Portuguese FCT Foundation (project UIDB/00066/2020), and the European Commission (project DiGiFoF). We also recognize the excellent contributions from Bruno Serras as a research fellow.

\section{References}

1. A. Balalaie, A. Heydarnoori, and P. Jamshidi. Microservices Architecture Enables DevOps: Migration to a Cloud-Native Architecture. IEEE Software, 33(3):42-52, May 2016. 
2. J. Han, S. Park, and J. Kim. Dynamic OverCloud: Realizing Microservices-Based IoTCloud Service Composition over Multiple Clouds. 9, 2020.

3. P. Hunt, M. Konar, F. P. Junqueira, and B. Reed. ZooKeeper: Wait-Free Coordination for Internet-Scale Systems. In Proceedings of the 2010 USENIX Conference on USENIX Annual Technical Conference. USENIX Association, 2010.

4. F. P. Junqueira and B. Reed. ZooKeeper: Distributed Process Coordination. O'Reilly Media, Inc., 1st edition, 2013.

5. F. P. Junqueira, B. Reed, and M. Serafini. Zab: High-Performance Broadcast for PrimaryBackup Systems. In Proceedings of the 2011 IEEE/IFIP 41st International Conference on Dependable Systems \& Networks, pages 245-256. IEEE Computer Society, 2011.

6. L. Lamport. Paxos Made Simple. ACM SIGACT News (Distributed Computing Column) 32, pages 51-58, December 2001

7. A. Luís Osório. Towards Vendor-Agnostic IT-System of IT-Systems with the CEDE Platform. In: Collaboration in a Hyperconnected World. PRO-VE 2016. IFIP AICT, vol 480. Springer, Cham. https://doi.org/10.1007/978-3-319-45390-3 42

8. A. Luís Osório, Adam Belloum, Hamideh Afsarmanesh, and Luís M. Camarinha-Matos. Agnostic Informatics System of Systems: The Open ISoS Services Framework. In: Collaboration in a Data-Rich World, pages 407-420. PRO-VE 2017. IFIP AICT, vol 506. Springer, Cham. https://doi.org/10.1007/978-3-319-65151-4_37

9. A. Luís Osório, Luís M. Camarinha-Matos, and Hamideh Afsarmanesh. ECoNet Platform for Collaborative Logistics and Transport. In: Risks and Resilience of Collaborative Networks. PRO-VE 2015. IFIP AICT, vol 463. Springer, Cham. https://doi.org/10.1007/978-3-319-24141-8_24

10. A. Luís Osório, Luís M. Camarinha-Matos, Hamideh Afsarmanesh, and Adam Belloum. On Reliable Collaborative Multimodal Services. In: Collaborative Networks of Cognitive Systems. PRO-VE 2018. IFIP AICT, vol 534. Springer, Cham. https://doi.org/10.1007/978-3-319-99127-6 26

11. A. Luís Osório, Luís M. Camarinha-Matos, Hamideh Afsarmanesh, and Adam Belloum. Liability in Collaborative Maintenance of Critical System of Systems. In: Boosting Collaborative Networks 4.0 . PRO-VE 2020, Valencia, Spain, November 23-25, 2020, Proceedings, volume 598 of IFIP AICT, pages 191-202. Springer International Publishing, 2020. https://doi.org/10.1007/978-3-030-62412-5 16

12. A. Luís Osório, Luís M. Camarinha-Matos, Tiago Dias, and José Tavares. Adaptive Integration of IoT with Informatics Systems for Collaborative Industry: the SITL-IoT Case. In: Collaborative Networks and Digital Transformation. PRO-VE 2019. IFIP AICT, vol 568. Springer, Cham. https://doi.org/10.1007/978-3-030-28464-0 5

13. Bruno Serras, Carlos Gonçalves, Tiago Dias, and A. Luís Osório. Synoptics of Things (SoT): An Open Framework for the Supervision of IoT Devices. In To appear in 5th International Young Engineers Forum on Electrical and Computer Engineering. IEEE Xplore digital library, 2021.

14. D. Taibi, V. Lenarduzzi, and C. Pahl. Continuous Architecting with Microservices and DevOps: A Systematic Mapping Study. CoRR, abs/1908.10337, 2019. 